\title{
Características psicométricas del Cuestionario Básico de Depresión en una muestra de adolescentes
}

\author{
Wenceslao Peñate ${ }^{1 *}$, Rosalía Bello ${ }^{1}$, Auxiliadora García ${ }^{1}$, Anna Rovella $^{2}$ y Tasmania del Pino-Sedeño ${ }^{1}$ \\ ${ }^{1}$ Dto. de Personalidad, Evaluación y TT.PP., Facultad de Psicología, Universidad de La Laguna (Tenerife, España) \\ 2 Universidad Nacional de San Luis (Argentina)
}

\begin{abstract}
Resumen: Se presentan datos sobre la validación del Cuestionario Básico de Depresión (CBD) en una muestra de adolescentes. El CBD es un inventario de 21 ítems que evalúa las áreas más representativas de la depresión, y que ha demostrado buenas propiedades psicométricas con muestras de adultos. El CBD fue administrado a 392 adolescentes (retest $n=60$ ), conjuntamente con el CDI y el STAIC. Los resultados favorecieron una solución unifactorial, con una buena consistencia interna y una elevada estabilidad temporal. La validez convergente con depresión fue elevada, pero también lo fue con ansiedad. El CBD muestra una mayor capacidad para detectar 'posibles casos' de depresión que 'posibles no casos'. Estos resultados se discuten en relación con la necesidad de corroborarlos con muestras clínicas.
\end{abstract}

Palabras clave: Evaluación de la depresión; cuestionarios; validación psicométrica; adolescentes.

\section{Introducción}

Los problemas emocionales representan los trastornos más prevalentes en la población infanto-juvenil, como se ha podido constatar en estudios multiculturales (Rescorla et al., 2007). Entre estos problemas emocionales destaca la depresión (usualmente acompaña de problemas de ansiedad). Datos similares se han encontrado en muestras españolas (López-Soler, Alcántara, Fernández, Castro y López Pina, 2010), que muestran cómo, atendiendo a una población infantojuvenil de riesgo, los niveles de depresión casi llegan a doblar a los problemas de ansiedad (López-Soler et al., 2012). Este impacto es más destacable por su vinculación al suicidio, tercera causa de muerte en estas edades (Ruble, Leon, GilleyHensley, Hess y Swartz, 2013; Young, 2012).

El Cuestionario Básico de Depresión (CBD, Peñate, 2001a) fue diseñado con la pretensión de aislar los síntomas genuinos de la depresión. El trastorno depresivo está caracterizado por un conjunto de síntomas muy dispersos (APA 2000; WHO, 1992), que incluyen distintos sistemas de respuesta (somáticos-neurovegetativos, cognitivo-afectivos, comportamentales...), con distintos grados de consolidación (v.g., la labilidad emocional y las actitudes estables frente a la vida), y con distintos niveles de complejidad (v.g., los sentimientos de tristeza y las quejas de mal funcionamiento cognitivo). A este panorama se une el hecho de que gran parte de esa sintomatología es compartida con frecuencia con otros trastornos, especialmente con los trastornos por el es-

* Dirección para correspondencia [Correspondence address]: Wenceslao Peñate Castro. Dto. Personalidad, Evaluación y TT.PP. Facultad de Psicología. Universidad de La Laguna - Campus de Guajara 38204 La Laguna - Tenerife- Islas Canarias (España). E-mail: wpenate@ull.es

\begin{abstract}
Title: Psychometric properties of the Basic Questionnaire for Depression with an adolescent sample.

Abstract: Data about the validation of the Basic Questionnaire for Depression (CBD, Cuestionario Básico de Depresión) with an adolescent sample, are presented. The CBD is a 21 -items inventory to assess the most representative areas of depression, and that has shown adequate psychometric properties with adult samples. The CBD was administered to 392 adolescents, (retest, $n=60$ ), jointly with the CDI and the STAIC. The results favoured an one-factor solution, with an elevated internal consistency and a high temporary stability. The convergent validity with depression was elevated, but also it was it with anxiety. The CBD shows a greater capacity to detect 'possible-cases' of depression than 'possible-not-cases'. These results are discussed in relation to the necessity to corroborate them with clinical samples.

Key words: Assessment of depression; questionnaires; psychometric validation; adolescents.
\end{abstract}

tado de ansiedad, como puso en evidencia el modelo tripartito para la ansiedad y la depresión (Clark y Watson, 1991).

Este estado de la cuestión podría ser una de las justificaciones de la existencia de un volumen importante de instrumentos sobre la depresión (Mulrow et al., 1995; Williams, Pignone, Ramirez y Stellato, 2002), cada uno de ellos acentuando determinados síntomas y procesos en sus contenidos. Instrumentos que, como ya constataron hace bastante tiempo Levitt y Lubin (1975), poseen entre sí escasas coincidencias.

En general las distintas escalas muestran unos buenos indicadores psicométricos en relación con su consistencia interna, su capacidad para diferenciar a personas con depresión frente a población no clínica, su validez convergente y su validez de tratamiento. Nezu, Ronan, Meadows y McClure (2008), han identificado 36 escalas para evaluar el fenómeno depresivo que reúnen dichos requisitos psicométricos (a estas escalas se pueden añadir otras 16 que evalúan los procesos depresivos en condiciones clínicas o poblaciones especiales, y 42 que evalúan constructos o procesos en relación con la depresión). Las dificultades han estado en su capacidad para el diagnóstico diferencial (especialmente con algunos trastornos de ansiedad, como se ha indicado) y su validez para discriminar entre distintos trastornos depresivos. Desde el punto de vista conceptual, se ha cuestionado la validez de constructo ante la dificultad de aislar un único componente depresivo.

El CBD consta de 21 ítems referidos a tres áreas típicamente depresivas: el afecto triste, la anhedonia y la baja autoestima (Peñate, 2001b y c), que conforman una estructura unifactorial. Esta escala ha mostrado su utilidad en algunos aspectos críticos como han sido la validez diferencial entre ansiedad y depresión (Peñate, Ibáñez y González, 2001; Viña, Herrero, Ibáñez, González y Peñate, 2004), su capacidad 
para discriminar entre distintos estados depresivos (Peñate y Almeida, 2001), o su validez de tratamiento (Peñate, Pitti, García y Perestelo, 2005).

Los contenidos recogidos en el CBD como los síntomas genuinos de la depresión ya han sido recogidos total o parcialmente en otras escalas, especialmente los referidos a los sentimientos de tristeza y a la anhedonia (v.g., el PHQ-9, Kroenke, Spitzer y Williams, 2001; HADS, Zigmond y Snaith, 1983; ST/DEP, Spielberger, Carretero-Dios, De los Santos-Roig y Buela-Casal, 2002a, 2002b). Incluso, desde otra perspectiva, se ha planteado que las dos primeras áreas del CBD, y por medio de dos ítems (un ítem de afecto triste y un ítem de anhedonia), serían suficientes para detectar el hecho depresivo (Kroenke, Spitzer y Williams, 2003). Sin embargo, cada una de las dos áreas por separado pierden capacidad predictiva (Corson, Gerrity y Dobscha, 2004). En este sentido, en el CBD se considera que las áreas/síntomas en cuestión (afecto triste, anhedonia y baja autoestima), si bien retóricamente pueden ser distinguidas, psicológicamente deben ser entendidas como una unidad (un único factor), con diferentes modos de expresión y que posiblemente den cuenta de la variedad de los procesos depresivos (Buckner, Joiner Jr., Pettit, Lewinsohn y Schmidt, 2008). Hasta ahora, la aplicación del CBD se ha hecho sobre muestras de adultos. Con el presente trabajo se pretende ofrecer datos de su validación con una muestra de adolescentes.

La evaluación de la depresión infanto-juvenil también dispone de escalas e inventarios (además de entrevistas estructuradas y escalas de calificación), suficientemente validados y que han mostrado su eficiencia clínica en la detección de los procesos depresivos infanto-juveniles (Williams, O'Connor, Eder y Whitlock, 2009), pero sobre los que todavía quedan criterios psicométricos por refinar (Ebesutani et al., 2010; Weber, 2009), lo que hace que todavía se sigan ofertando nuevas escalas (v.g., Pietsch et al., 2011), o adaptando otras (v.g., Ganguly et al., 2013).

Estas escalas e inventarios rastrean contenidos típicos en relación con el proceso depresivo, añadiendo los elementos más peculiares de la depresión infanto-juvenil (irritabilidad, impulsividad, cambios comportamentales, autodesprecio...). A pesar de que la producción científica en este campo en el estado español es mayoritariamente teórica (Díez Zamorano, 2003), se cuenta con buenas adaptaciones de algunos de estos instrumentos (Inventario de Depresión Infantil, del Barrio y Carrasco, 2004; Escala de Depresión Adolescente de Reynolds, Figueras-Masip, Amador-Campos y Peró-Cebollero, 2008; Cuestionario de Depresión infantil, Lang y Tisher, 1983).

El proponer una adaptación del CBD a la población adolescente, aun cuando se dispone de instrumentos específicos para ello, se debe al interés por conocer si esa estructura formada por tres áreas principales de la depresión también se puede sostener en la depresión infanto-juvenil. Como ha ocurrido con muestras adultas, el propósito se fundamenta en el concepto de validez incremental. Más específicamente, en las tres concepciones propuestas por Hunsley y Meyer (2003): capacidad evaluativa (mejora en la predicción de un cri- terio clínico relevante); la capacidad inferencial (en qué medida una nueva prueba permite hacer juicios diagnósticos y tomas de decisiones más precisas); y la validez de nuevas medidas (en qué medida la propuesta de una nueva prueba sobre algún fenómeno psicológico aporta información significativa sobre las pruebas existentes). Secundariamente, se trataría de proporcionar una prueba que permitiera una comparación directa de los niveles de depresión en estudios longitudinales, una vez que parece constatarse que la depresión es más transitoria que otros trastornos emocionales (Prenoveau et al., 2011).

En ese sentido, hemos tratado de identificar los contenidos más precisos de la depresión y hemos ido añadiendo datos de validación que mejoraran la capacidad predicativa y la toma de decisiones por medio de una nueva medida. Lo que se trataría ahora es verificar si esos hallazgos también son aplicables a otras muestras, donde la depresión posee sus propias características. Ese es el objetivo general de este trabajo: aportar información psicométrica sobre el comportamiento del CBD en una muestra de adolescentes.

\section{Método}

\section{Participantes}

Trescientos noventa y dos adolescentes de dos institutos de la comarca norte de la isla de Tenerife. El 53\% eran chicos. La edad media fue de 13.76 años (rango de 12 a 16). Por niveles educativos, $37.3 \%$ cursaban primero de la ESO, $35.7 \%$ segundo; y $27 \%$ tercero. En el retest participaron 60 , con una edad media de 14 años. El $40 \%$ eran de $1^{\circ}$ de la ESO, 36.7 de $2^{\circ}$, y 23.3 de $3^{\circ}$. En este caso la mayoría, el $68.3 \%$, eran chicas (se comenta en el procedimiento).

\section{Instrumentos}

Además del CBD, se administraron dos cuestionarios más: el Inventario de Depresión Infantil y el Inventario Ansiedad Estado-Rasgo para niños.

- Cuestionario Básico de Depresión (Peñate, 2001a). Como se ha indicado, el CBD consta de 21 ítems referidos a tres áreas del estado de ánimo: afecto triste, anhedonia y baja autoestima. El formato de respuestas es de cuatro alternativas, de acuerdo con el curso temporal de la depresión: (0) ausencia (si el síntoma no ha estado presente o su duración ha sido inferior a dos semanas); (1) semanas (si el síntoma ha estado presente más de dos semanas); (2) meses (si ha estado presente durante meses); y (3) años (si lleva años con ese síntoma). Tal y como se señala en la introducción, el CBD ha mostrado buenos ajustes psicométricos con muestras adultas. Los datos clínicos disponibles para muestras de adultos señalan las siguientes categorías según la puntuación: de 0 a 19, no depresión; de 20 a 29, depresión leve; de 30 a 39, depresión moderada; y 40 o más, depresión grave.

- Inventario de Depresión Infantil (CDI, Kovacs, 1992). El CDI tiene como finalidad la evaluación de la sintomatología de- 
presiva y está compuesto por 27 ítems, cada uno de ellos enunciados en tres frases que recogen la distinta intensidad o frecuencia de su presencia en el niño o adolescente (de 7 a 15 años), mostrando una adecuada capacidad para identificar los estados depresivos (Levitt, Saka, Romanelli y Hoagwood, 2007). Además de una escala general, posee dos subescalas, disforia (humor depresivo, tristeza, preocupación...) y autoestima negativa (juicios de ineficacia, fealdad, maldad...). Los datos psicométricos en la adaptación española (del Barrio y Carrasco, 2004), ha mostrado buenos índices psicométricos, tanto en su validez interna como en la validez externa.

- Inventario de Ansiedad Estado-Rasgo para niños (STAIC, Spielberger, 1973). El STAIC es un inventario de autoevaluación de la ansiedad estado y la ansiedad rasgo, de acuerdo con el modelo de Charles Spielberger. Se administra a niños de edades comprendidas entre 9 y 16 años. En nuestro caso hemos pasado sólo la escala que mide la ansiedad rasgo, que comprende 20 elementos con los que el participante puede indicar "como se siente en general" en una escala de respuestas de tres alternativas. La adaptación española (Seisdedos, 1990), muestra buenos ajustes psicométricos. En general es una escala con una mayor especificidad que sensibilidad (Levitt et al., 2007).

\section{Procedimiento}

Los datos se recogieron en dos institutos de enseñanza secundaria, ubicados en la comarca norte de la isla de Tenerife. El contacto con la dirección y profesorado fue una gestión personal de los autores de este artículo. Participaron estudiantes de $1^{\circ}$ a $3^{\circ}$ ESO. Se pidió el consentimiento del profesorado y del alumnado para llevar a cabo el pase de pruebas. Al alumnado se le daba la opción de presentarle sus resultados individualmente, si así lo hacían explícito en el cuadernillo que contenía sus datos socio-demográficos y las pruebas. Estos resultados se les comentaba alrededor de los tres meses. En esos momentos se aprovechaba para que, los que asistían a conocer sus datos (60 chicos y chicas), cumplimentaran de nuevo el CBD (retest).

El pase de pruebas se hizo de manera grupal, en las propias clases. Se les daba una instrucción general (confidencialidad de la información, sinceridad, leer detenidamente las instrucciones, frases o preguntas, y tratar de no dejar ninguna pregunta sin contestar). Además, se les indicaba que en cada página disponían de instrucciones específicas de cómo cumplimentar cada una de las pruebas, quedando los evaluadores a disposición de ellos para aclarar las dudas que tuvieran. No se les dio tiempo de finalización, aunque no llegaban a la hora (una clase completa).

Una vez que se grabaron los datos, se llevaron a cabo los análisis estadísticos (paquete SPSS 15.0 y AMOS 6.0). Los análisis consistieron en análisis factoriales del CBD y análisis correlacionales y de contraste de medias para analizar su validez convergente y diferencial. Finalmente se llevaron a cabo análisis para determinar su sensibilidad y especificidad.

\section{Resultados}

\section{Estructura factorial y fiabilidad}

En primer lugar se llevó a cabo un análisis factorial exploratorio mediante extracción por componentes principales y rotación oblicua, ya que se partía de que los posibles factores a extraer estarían relacionados entre sí. El coeficiente KMO encontrado fue de .85 y la prueba de esfericidad de Bartlett obtuvo un de $\chi^{2}=2415.92$, altamente significativo para 210 grados de libertad $(p \leq .0001)$. Estos datos avalan los supuestos necesarios para llevar a cabo el análisis factorial. La solución obtenida se recoge en la Tabla 1.

Como puede observarse, se obtuvo una solución trifactorial, con un gran peso del primer factor. Sin embargo, tomando como criterio saturaciones $\geq .30$, salvo el ítem 15 ("me veo una persona atractiva"), el resto obtiene saturaciones $\geq .30$ en el primer factor. El porcentaje de varianza explicado de ese primer factor es claramente mayor que el de los otros dos factores. El segundo factor tiene como ítem marcador al 18 ("nada me interesa") y da cuenta de una suerte de componente de estado de ánimo positivo con elementos circunstanciales de angustia y culpabilidad. El tercer factor tiene como ítem marcador al 15 ya comentado y posee una estructura más dispar desde el punto de vista interpretativo. Las correlaciones encontradas entre los tres factores fueron bajas, a pesar de utilizar una rotación oblicua (-.14 entre el primer y segundo factor; .06 entre el primero y el tercero; y -.03 entre el segundo y el tercero). Asimismo, los coeficientes de consistencia interna ( $\alpha$ de cronbach) fueron dispares: .87 para el primer factor; .67 para el segundo; y .38 para el tercero.

Estos datos nos estarían indicando que, en la práctica, la solución era una solución monofactorial, ya que el primer factor era el único que presentaba una cierta robustez psicométrica. Para contrastarlo se llevó a cabo un análisis factorial confirmatorio (SPSS, AMOS 6.0). No se estimó la estructura trifactorial porque se recomienda un mínimo de cuatro indicadores por factor y los factores segundo y tercero sólo contenían dos ítems/indicadores (ítems 1 y 18, factor segundo; ítems 15 y 19, factor tercero).

Tomando en cuenta esas restricciones, sólo se pudo someter a validación el modelo unifactorial. El método de estimación utilizado fue el de máxima verosimilitud. En la Tabla 2 se resumen los coeficientes e índices obtenidos.

De acuerdo con estos indicadores, los datos favorecerían la idea de esa solución monofactorial, aunque el CFI (Comparative Fit Index) no llegó a alcanzar una puntuación de .95.

Con respecto a la estabilidad temporal, a los tres meses se volvió a administrar el CBD a aquellos estudiantes que acudieron a conocer sus resultados $(n=60)$. La $\mathrm{r}_{\mathrm{xy}}$ obtenida fue de .76, altamente significativa $(p \leq .01)$. 
Tabla 1. Estructura factorial del CBD en una muestra de adolescentes $(n=392)$. Extracción con componentes principales y rotación oblicua.

\begin{tabular}{|c|c|c|c|c|}
\hline \multirow{2}{*}{\multicolumn{2}{|c|}{ Ítems }} & \multicolumn{3}{|c|}{ Factores } \\
\hline & & 1 & 2 & 3 \\
\hline 1. Me considero una persona alegre. & & -.30 & .39 & .32 \\
\hline 2. Me siento culpable. & & .53 & .32 & .25 \\
\hline 3. Me siento fracasado o fracasada. & & .65 & .15 & -.06 \\
\hline 4. Me siento infeliz. & & .63 & .16 & .17 \\
\hline 5. Me cuesta mucho vivir. & & .67 & .00 & .31 \\
\hline 6. Me siento triste. & & .66 & .34 & .11 \\
\hline 7. Me siento angustiado o angustiada. & & .62 & .44 & .14 \\
\hline 8. Me siento culpable de lo que ocurre a los demás. & & .34 & .31 & .27 \\
\hline 9. Me considero una persona débil. & & .47 & .29 & -.34 \\
\hline 1. Siento miedo ante la vida. & & .59 & .24 & -.17 \\
\hline 11. Me siento solo o sola. & & .58 & .06 & -.09 \\
\hline 12. Pienso que soy una persona sin suerte. & & .51 & -.09 & -.28 \\
\hline 13. Creo que los demás valen más que yo. & & .66 & -.10 & -.17 \\
\hline 14. Me gustaría ser distinto o distinta a como soy. & & .57 & -.07 & -.19 \\
\hline 15. Me veo una persona atractiva. & & -.19 & -.03 & .66 \\
\hline 16. Veo más defectos que virtudes en mi persona. & & .67 & -.22 & -.02 \\
\hline 17. Creo que no valgo para nada. & & .64 & -.28 & -.21 \\
\hline 18. Nada me interesa. & & .25 & -.49 & .21 \\
\hline 19. No disfruto con las cosas que antes disfrutaba. & & .41 & -.34 & .45 \\
\hline 2. Mi motivación general es mínima o nula. & & .57 & -.44 & .30 \\
\hline 21. Me siento incapaz de divertirme y pasarlo bien. & & .42 & -.40 & -.06 \\
\hline & Valor propio & 6.09 & 1.70 & 1.52 \\
\hline & $\%$ de varianza & 29.00 & 8.12 & 7.22 \\
\hline & $\%$ de varianza rotada & 53.05 & 14.81 & 13.24 \\
\hline
\end{tabular}

Tabla 2. Principales coeficientes e índices obtenidos del análisis factorial confirmatorio $(n=392)$.

\begin{tabular}{|lllllll|}
\hline MODELO & $G L$ & $\chi^{2}$ & $S R M R$ & AGFI & PGFI & CFI \\
UN FACTOR & 189 & $939^{*}$ & .05 & .91 & .33 & .93 \\
\hline
\end{tabular}

\section{$* p \leq .01)$}

Nota: GL $=$ grados de libertad; $\chi 2=$ chi cuadrado; SRMR $=$ Standardized Root Mean Square Residual; GFI = Goodness of Fit Index; AGFI = Adjusted Goodness of Fit Index; PGFI = Parsimony GFI; CFI = Comparative Fit Index.

\section{Validez convergente y discriminante}

Con respecto a la validez convergente, se llevaron a cabo análisis correlacionales entre el CBD, la medida de depresión del CDI (puntuación total, y con las subescalas de disforia y baja autoestima) y la medida de ansiedad con el STAIC. Con el CDI se obtuvo una $r_{x y}=.73$; con la subescala de disforia una $r_{x y}=.70$; y con la subescala de baja autoestima una $r_{x y}=$ .58. Todas estas correlaciones fueron significativas $(p \leq .01)$. Sin embargo, la correlación con el STAIC también fue elevada y considerable $\left(r_{x y}=.70 ; p \leq .01\right)$.

Tomando en cuenta las variables sociodemográficas, se obtuvo una diferencia ligeramente significativa entre chicos $(M=10.25 ; D T=7.34)$ y chicas $(M=12.28 ; D T=8.67)$, con $t_{374}=2.43 ; \mathrm{p} \leq .02$, siendo la puntuación de las chicas más elevadas en depresión, aunque con un tamaño del efecto bajo pequeño ( $d$ de Cohen $=.25$ ) .

Con respecto a los grupos de edades (de 12 a 16 años) se observó, mediante un análisis de varianza, una ligera significación $\left(F_{4,370}=2.72 ; p \leq .03\right)$, con un escaso tamaño del efecto $\left(\eta^{2}=.03\right)$. Las diferencias mostraban un incremento lineal a medida que se ascendía en la edad. Sin embargo, a pesar de ese incremento, el contraste de Bonferroni no ofreció ninguna diferencia significativa entre pares de edades.

Por lo que se refiere a la validez discriminante entre diferentes grupos clínicos, tomando los puntos de corte del CDI como criterio clínico (no depresión, $n=320$; depresión leve, $n=25$; y depresión severa, $n=11$ ), se llevó a cabo un nuevo contraste para conocer si esos tres niveles discriminaban en relación con la puntuación en el CBD. La $F$ obtenida para 2 y 353 grados de libertad fue de 108.8, altamente significativa $(\phi \leq .001)$, con un tamaño del efecto apreciable $\left(\eta^{2}=.38\right)$. Los contrastes por pares de grupo (Bonferroni) también mostraron diferencias significativas entre todos los emparejamientos. Estas diferencias son claramente apreciables en la Figura 1.

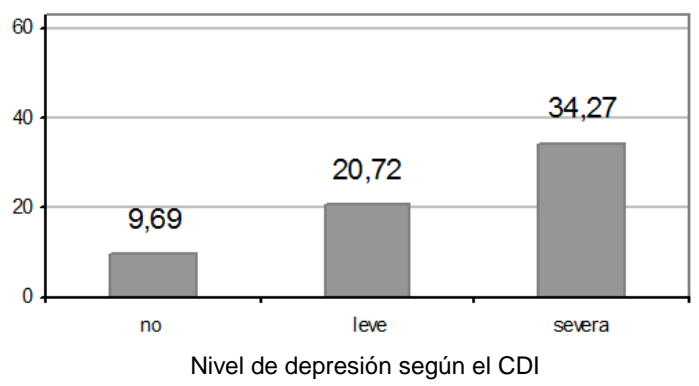

Figura 1. Puntuaciones medias en el cuestionario básico de depresión (CBD) de los tres grupos clínicos, según los criterios del Inventario de Depresión Infantil (CDI). 


\section{Capacidad de clasificación correcta depresión-no depresión}

Finalmente para conocer la capacidad de CBD para clasificar correctamente entre adolescentes con depresión (caso) y sin depresión (no caso), se llevó a cabo un análisis de curva de ROC, tomando como criterio las puntuaciones en el CDI. Se dicotomizó a la muestra de acuerdo a posible caso de depresión en el CDI (depresión leve o depresión severa), frente a posible no caso (no depresión), y también de acuerdo a ese criterio para el CBD (caso = depresión leve, moderada y grave; no caso $=$ no depresión).

Las odds-ratio obtenidas (tomando el CDI como criterio) fueron las siguientes: razón de verosimilitud positiva = 15.25; razón de verosimilitud negativa $=.41$; lo que nos vino a indicar una excelente capacidad para identificar casos de depresión por parte del CBD, pero mayores dificultades para identificar posibles no casos.

\section{Discusión}

El CBD es uno de los variados cuestionarios, inventarios y escalas desarrollados para evaluar el nivel de depresión. La mayor especificidad de este cuestionario consiste en que sólo evalúa síntomas depresivos en relación con tres áreas que, tentativamente, serían las áreas genuinas de la depresión, a saber, afecto triste, anhedonia y baja autoestima (Peñate, 2001 b y c). En este sentido, una de las mayores fortalezas de este cuestionario estaría en su capacidad para discriminar los procesos depresivos de otros procesos, especialmente con los procesos de ansiedad (Peñate et al., 2001; Viña et al., 2004).

Los datos de validación del CBD se han obtenido con muestras adultas. Con el presente trabajo se ha pretendido conocer cómo se comportaba este cuestionario con una muestra de adolescentes, a sabiendas de que los procesos depresivos infanto-juveniles difieren cualitativamente de la depresión en la etapa adulta.

Los resultados expuestos muestran que la aplicación del CBD a una muestra de adolescentes obtiene propiedades psicométricas comparables a las obtenidas en la muestras de adultos, especialmente en su estructura factorial. De esta manera los 21 ítems que representan al afecto triste, la anhedonia y la baja autoestima, obtienen en la práctica una solución unifactorial, con una adecuada consistencia interna y una elevada estabilidad temporal a los tres meses. Esta solución muestra una buena validez convergente y criterial con las puntuaciones en el CDI. Sin embargo, algunos resultados son más específicos y se separan de los obtenidos con muestras adultas: la estrecha relación con la ansiedad y las diferencias por sexo y edad.

En referencia a la relación con la ansiedad, el coeficiente de correlación entre el CBD y una medida como el STAIC es estimable y significativo. Esta elevada convergencia con una medida de ansiedad de alguna manera cuestionaría su carácter genuino de la depresión. Evidentemente así puede entenderse, pero también es cierto que la medida ansiedadrasgo del cuestionario de Charles Spielberger es una medida muy convergente con medidas de depresión, a veces incluso mayor que la que obtienen las medidas de depresión entre sí (v.g., Spielberger, Buela-Casal, Agudelo, Carretero-Dios y Santolaya, 2005). De hecho, si se analizan los contenidos de la escala de ansiedad se puede observar que contiene elementos prototípicos de depresión (alegría/tristeza, cansancio, indecisión, lloros...), y pueden perfectamente pasar por una escala de medición de la afectividad negativa (Agudelo, Buela-Casal y Spielberger, 2007). Afectividad que representa el elemento común entre ansiedad y depresión (Anderson y Hope, 2008). En este sentido, es esperable entonces que la validación convergente sea elevada también con el STAIC. Lo que está pendiente entonces es conocer las relaciones entre el CBD y una escala de ansiedad más específica.

Las diferencias encontradas entre chicos y chicas (las chicas tienden a puntuar ligeramente más), no son datos que se correspondan con el CBD en adultos, pero está dentro de la lógica epidemiológica de la depresión (todavía la depresión es un trastorno feminizado, ya que la proporción mujeres con depresión suele ser más elevada que la de los hombres), y en ese sentido, no es un resultado anómalo. Quizás las áreas que evalúa el $\mathrm{CBD}$ sean áreas más sensibles en chicas que en chicos a esas edades, pero que se van normalizando a medida que se asciende en la edad. De la misma manera que la puntuación media asciende en general con edad (como si hubiera una mayor sensibilización o reconocimiento de la sintomatología depresiva con el paso de los años), pudiera ocurrir que esa sensibilización tiene ritmos distintos entre chicos y chicas. En todo caso, el que las chicas puntúen más que los chicos y que la puntuación se incremente con la edad son resultados esperables en la medición de la depresión infanto-juvenil (v.g., Figueras-Masip et al., 2008; Zubeidat, Fernández-Parra, Ortega, Vallejo y Sierra, 2009). Es posible que este incremento de la puntuación a medida que se asciende en la edad también se deba una mayor intensidad de los adolescentes con la sintomatología depresiva, especialmente con los sentimientos de tristeza (Siverio y GarcíaHernández, 2007). Sin embargo, estos datos se contradicen con los hallados por Bernaras, Jaureguizar, Soroa, Ibabe y de las Cuevas (2013), donde se daba una interacción entre género y edad: mientras la puntuación disminuía con la edad en chicos, aumentaba en las chicas. Quizás pueda ser explicado porque estos autores toman cinco grupo de edades (de 8 a 12 años), más tempranas que las tomadas en este estudio (a partir de los 12 años).

Finalmente la capacidad clasificatoria del CBD cuestiona su capacidad para identificar "no casos" de depresión. Este hecho puede deberse en parte a un efecto del criterio, tomando como tal a la puntuación en el CDI, ya que formalmente el CDI no contempla la categoría de depresión moderada (sólo leve y severa). Posiblemente si se hubiera creado esa categoría, algunos participantes leves y algunos severos caerían dentro de esa categoría; y, por otro, la moderada capacidad para identificar "no casos" (la razón de verosimilitud 
negativa indica un porcentaje de posibles errores del $40 \%$, lo que estaría indicando que el CBD puede estar "produciendo" casos), puede estar indicando también un problema con el criterio diagnóstico utilizado.

Sin querer entrar en una disquisición más profunda sobre cierta cuantitofrenia en la elaboración de tests (MateoGarcía, 2011), lo cierto es que el CDI en sí mismo no es un criterio diagnóstico para el trastorno depresivo. El tomarlo como criterio era un modo de validar el CBD, pero no se puede decir en sentido estricto que las personas con categorías leves o severas en el CDI posean un diagnóstico de trastorno depresivo. $\mathrm{Ni}$ tampoco se puede decir que todos los

\section{Referencias}

Agudelo, D., Buela-Casal, G., \& Spielberger, C. (2007). Ansiedad y depresión: el problema de la diferenciación a través de los síntomas. Salud Mental, 30 (2), 33-41.

Anderson, E. R., \& Hope, D. A. (2008). A review of the tripartite model for understanding the link between anxiety and depression in youth. Clinical Psychology Review, 28, 275-287.

American Psychiatric Association (2000). Diagnostic and Statistical Manual of Mental Disorders, Fourth Edition, Text Revision. Washington: Author.

Bernaras, E., Jaureguizar, J., Soroa, M., Ibabe, I., \& de las Cuevas, C. (2013). Evaluación de la sintomatología depresiva en el contexto escolar y variables asociadas. Anales de psicología, 29, 131-140.

Buckner, J. D., Joiner Jr., T. E., Pettit, J. W., Lewinsohn, P. M., \& Schmidt, N. B.(2008). Implications of the DSM's emphasis on sadness and anhedonia in major depressive disorder. Psychiatry Research, 159 (1-2), 25-30.

Clark, L. A., \& Watson, D. (1991). Tripartite model of anxiety and depression: Psychometrics evidence and taxonomic implications. Journal of $A b$ normal Psychology, 3, 316-336.

Corson, K., Gerrity, M. S., \& Dobscha, S. K. (2004). Screening for depression and suicidality in a VA primary care setting: 2 items are better than 1 item. The American Journal of Managed Care, 10, 839-845.

del Barrio, V., \& Carrasco, M. A. (2004). Inventario de Depresión Infantil. Madrid: TEA Ediciones.

Díez Zamorano, M. A. (2003). Análisis bibliométrico sobre depresión infantil en España. International Journal of Clinical and Health Psychology, 1, 645653 .

Ebesutani, C., Chorpita, B. F., Higa-McMillan, C. K., Nakamura, B. J., Regan, J. \& Lynch, R. E. (2010). A Psychometric Analysis of the Revised Child Anxiety and Depression Scales-Parent Version in a School Sample. Journal of Abnormal Child Psychology 39, 173-185.

Figueras-Masip, A., Amador-Campos, J. A., \& Peró-Cebollero, M. (2008). Características psicométricas de la Reynolds Adolescent Depression Scale en población comunitaria y clínica. International Journal of Clinical and Health Psychology, 8, 247-266.

Ganguly, S., Samanta, M., Roy, P., Chatterjee, S., Kaplan, D. W., \& Basu, B. (2013). Patient Health Questionnaire-9 as an effective tool for screening of depression among Indian adolescents. Journal of Adolescent Health, 52, 546-551.

Hunsley, J., \& Meyer, G. J. (2003). The incremental validity of psychological testing and assessment: Conceptual, methodological, and statistical issues. Psychological Assessment, 15, 446-455.

Kovacs, M. (1992). Children's Depression Inventory. Toronto: Multi-Health Systems.

Kroenke, K., Spitzer R. L., \& Williams, J. B. (2001).The PHQ-9: Validity of a brief depression severity measure. Journal of General Internal Medicine, 16, 606-613.

Kroenke, K., Spitzer, R. L., \& Williams, J. B. (2003). The Patient Health Questionnaire-2: Validity of a two-item depression screener. Medical Care, 41, 1284-1292.

Lang, M., \& Tisher, M. (1983). Cuestionario de depresión para niños. Madrid: TEA Ediciones. "no casos" realmente lo sean. Esto nos lleva a la necesidad de que en el futuro habría que observar el comportamiento del CBD en muestras clínicas infanto-juveniles con depresión.

En definitiva, el CBD se comporta adecuadamente de manera estructural, posee indicadores psicométricos con cierta bondad, pero quedan por resolver algunos problemas. El más importante (porque tiene que ver con la razón de ser del CBD) es su capacidad para discriminar entre depresión y otros problemas, especialmente con los problemas de ansiedad. La necesaria validación con muestras clínicas podrá añadir más información en este sentido.

Levitt, E., \& Lubin, B. (1975). Depression. Concepts, controversies, and some new facts. Nueva York: Springer.

Levitt, J. M., Saka, N., Romanelli L. H., \& Hoagwood, K. (2007). Early identification of mental health problems in schools: The status of instrumentation. Journal of School Psychology, 45, 163-191.

López-Soler, C., Fernández, M. V., Prieto, M., Alcántara, M. V., Castro, M., \& López-Pina, J. A. (2012). Prevalencia de las alteraciones emocionales en una muestra de menores maltratados. Anales de psicología, 28, 780-788.

López-Soler, C., Alcántara, M. V., Fernández, V., Castro, M. \& López Pina, J. A. (2010). Características y prevalencia de los problemas de ansiedad, depresión y quejas somáticas en una muestra clínica infantil de 8 a 12 años, mediante el CBCL (Child Behavior Checklist). Anales de Psicología, 26, 325-334.

Mateo-García, M. A. (2011). Sobre un método comúnmente utilizado para la puntuación de tests en la evaluación del rendimiento educativo. Anales de Psicología, 27, 249-255.

Mulrow, C. D., Williams, J. W., Gerety, M. B., Ramirez, G., Montiel, O. M. \& Kerber, C. (1995). Case-finding instruments for depression in primary care settings. Annals of Internal Medicine, 122, 913-921.

Nezu, A. M., Ronan, G. F., Meadows, E. A., \& McClure, K. S. (2008). Practitioner's Guide to Empirically Based Measures of Depression. Nueva York: Springer.

Peñate, W. (2001a). Presentación de un cuestionario básico para evaluar los síntomas genuinos de la depresión. Introducción. Análisis y Modificación de Conducta, 27, 679-731

Peñate, W. (2001b). Primer estudio: El cuestionario básico de depresión (CBD): Primeros datos de validación. Análisis y Modificación de Conducta 27, 733-761.

Peñate, W. (2001c). Cuarto estudio: Revisión del cuestionario básico de depresión: Afecto triste, anhedonia y baja autoestima. Análisis y Modificación de Conducta, 27, 799-809.

Peñate, W., \& Almeida, A. (2001). Quinto estudio: Validez discriminante de CBD-rev entre distintos tipos de trastornos depresivos. Análisis y Modificación de Conducta, 27, 811-829.

Peñate, W., Ibáñez, I., \& González, M. (2001). Sexto estudio: La estructura final del CBD-rev. Implicaciones para el diagnóstico diferencial con los trastornos de ansiedad. Análisis y Modificación de Conducta, 27, 831-849.

Peñate, W., Pitti, C. T., García, A., \& Perestelo, L. (2005). La validez de tratamiento del cuestionario básico de depresión. Primeros datos. Análisis y Modificación de Conducta, 31, 379-403.

Pietsch, K., Allgaier, A-K., Frühe, B., Rohde, S., Hosie, S., Heinrich, M., \& Schulte-Körne, G. (2011). Screening for depression in adolescent paediatric patients: Validity of the new Depression Screener for Teenagers (DesTeen). Journal of Affective Disorders 133, 69-75.

Prenoveau, J. M., Craske, M .G., Zinbarg, R. E., Mineka, S., Rose, R. D., \& Griffith. J. W. (2011). Are anxiety and depression just as stable as personality during late adolescence? Results from a three-year longitudinal latent variable study. Journal of Abnormal Psychology, 120, 832-843.

Rescorla, L., Achenbach, T., Ivanova, M., Dumenci, L., Almqvist, F., Bilenberg, N., ...Verhulst, F. (2007). Behavioral and Emotional Problems 
Reported by Parents of Children Ages 6 to 16 in 31 Societies. Journal of Emotional and Behavioral Disorders, 15, 130- 142.

Ruble, A. E., Leon, P. J., Gilley-Hensley, L., Hess, S. G., \& Swartz, K. L. (2013). Depression knowledge in high school students: Effectiveness of the adolescent depression awareness program. Journal of Affective Disorders, 150, 1025-1030.

Seisdedos, N. (1990). STAIC Cuestionario de Autoevaluación Ansiedad Estado/Rasgo en niños. Madrid: TEA Ediciones.

Siverio, M. A., \& García-Hernández, M. D. (2007). Autopercepción de adaptación y tristeza en la adolescencia: La influencia del género. Anales de Psicología, 23, 41-48.

Spielberger, C. (1973). STAIC, State-Trait Anxiety Inventory for Children. Palo Alto, CA: Consulting Psychologists Press.

Spielberger, C., Buela-Casal, G., Agudelo, D., Carretero-Dios, H., \& Santolaya, F. (2005). Análisis de la validez convergente y discriminante de la versión experimental castellana del Cuestionario de Depresión EstadoRasgo (ST-DEP). Actas Españolas de Psiquiatría, 33, 374-382.

Spielberger, C., Carretero-Dios, H., De los Santos-Roig, M., \& Buela-Casal, G. (2002a). Spanish experimental version of the State-Trait depression Questionnaire (ST-DEP: State subscale (S-DEP). International Journal of Clinical and Health Psychology, 2, 51-69.

Spielberger, C., Carretero-Dios, H., De los Santos-Roig, M., \& Buela-Casal, G. (2002b). Spanish experimental version of the State-Trait depression Questionnaire (ST-DEP): Trait sub-scale (T-DEP). International Journal of Clinical and Health Psychology, 2, 71-89.

Viña, C., Herrero, M., Ibáñez, I., González, M., \& Peñate, W. (2004). El modelo tripartito: Relaciones conceptuales y empíricas entre ansiedad, depresión y afecto negativo. Revista Latinoamericana de Psicología, 36, 289304

Weber, S. (2009). Results of psychometric testing of the RADS-2 with school-based adolescents seeking assistance for sexual orientation and gender identity concerns. Part 2: Research brief. Journal of Child and Adolescent Psychiatric Nursing, 22, 126-131.

WHO (1992). ICD-10: International Statistical Classification of Diseases and Related Health Problems. Geneve: Author.

Williams, J. W., Pignone, M., Ramirez, G. \& Stellato, C. P. (2002). Identifying depression in primary care: A literature synthesis of case-finding instruments. General Hospital Psychiatry, 24, 225-237.

Williams, S. B., O'Connor, E. A., Eder, M., \& Whitlock, E. P. (2009). Screening for child and adolescent depression in primary care settings: a systematic evidence review for the US Preventive Services Task Force. Pediatrics, 123, e716-e735.

Young, C. C. (2012). Screening for Depression in Adolescents. The Journal for Nurse Practitioners, $8,73-74$.

Zigmond, A. S., \& Snaith, R. P. (1983). The Hospital Anxiety and Depression Scale. Acta Pschyatrica Scandinavica, 67, 361-370.

Zubeidat, I., Fernández-Parra, A., Ortega, J., Vallejo, M. A., \& Sierra, J. C. (2009). Características psicosociales y psicopatológicas en una muestra de adolescentes españoles a partir del Youth self-report/11-18. Anales de Psicología, 25, 60-69.

(Articulo recibido: 31-08-2011; revisado: 2-9-2013; aceptado: 26-09-2013) 